\title{
How to Win the House: NBA Odds Analysis and Predictions
}

\author{
Wenpeng Di', Bin Yuan², Haiyi Wen ${ }^{3}$ \\ ${ }^{1}$ College of Letters \& Science, UC Santa Barbara, Santa Barbara, CA, USA \\ ${ }^{2}$ School of Traffic and Transportation, Chongqing Jiaotong University, Chongqing, China \\ ${ }^{3}$ Wuhan Britain-China School, Wuhan, China \\ Email: 651112011@qq.com
}

How to cite this paper: Di, W. P., Yuan, B., \& Wen, H. Y. (2021). How to Win the House: NBA Odds Analysis and Predictions. Open Journal of Social Sciences, 9, 177-187. https://doi.org/10.4236/jss.2021.92013

Received: December 11, 2020

Accepted: February 16, 2021

Published: February 19, 2021

Copyright (C) 2021 by author(s) and Scientific Research Publishing Inc. This work is licensed under the Creative Commons Attribution International License (CC BY 4.0).

http://creativecommons.org/licenses/by/4.0/

\begin{abstract}
In recent years, odd-making systems, from automated bookmakers and computer casino software to sophisticated market-research tools for betting and investment, have increasingly sought to capture the most accurate spread of outcome probabilities, and so betting odds have been increasingly dominated by algorithms for generating the spread (Spann \& Skiera, 2009). This study analyzes NBA betting spreads and odds in the 2016-2017 season to investigate the degree to which these odds have succeeded in capturing the spread of the game in real-life. In this paper, models are developed by the method of multiple linear regression to predict the results of the game based on the team's past performance. The model can help bookmakers optimize their algorithms. By reading this paper can also help the normal players with weak statistical base understand how the game works.
\end{abstract}

\section{Keywords}

NBA Ranking, Linear Regressions, Dummy Variable

\section{Introduction}

Sports betting has exploded over the last few years (Humphreys, Paul, \& Weinbach, 2013), with the total market value of this complex enterprise peaking at over $\$ 85$ billion in 2019 and projected to exceed $\$ 100$ billion by 2022 . However, the most challenging aspect about betting is the perennial question: how do bettors win against the house? Bettors look at spreadsheets, forms of the teams/players, and other types of information to predict the outcome of the game but are left with the dilemma of how to best make a rational decision on which strategy to employ and to the extent to which each is best supported by 
information available to maximize their chances of winning (Golec \& Tamarkin , 1995).

The purpose of this paper is to identify a possible research model for predicting potential outcomes of a sport game based on past odds proposed by sports handicappers and the true spread of the game. The model incorporates the linear regression process used to establish a model prediction based upon historical odds and the distribution of possible wins and losses. The dataset used in this study consists of all games in the NBA 2016-2017 season with respective odds and spread for each game as well as the odds distributions of the teams competing during the season.

\section{Research Method}

We are building on all the NBA games data of a certain season to mainly compare the strength of each team, make some prediction and demonstrate the accuracy of prediction.

The tools we are using are $\mathrm{R}$ language, matrix, modeling by using multiple linear regression, and so on. Firstly, we import the data from a website and delete useless part of it to make the data clear and available for next research. Then we applied dummy variables, which can show the two sides in matrixes clearly. By applying multiple linear regression models in $\mathrm{R}$ language, we can transform the team strength of the team into intuitive numbers, which will be more readable. As we expect, we can put the data in a regression model, the larger coefficients mean greater team strength. We analyze the factors which have influence on games' total scores and difference scores. Finally, we draw a conclusion.

$$
y_{i}=\beta_{0}+\beta_{1} x_{i 1}+\beta_{2} x_{i 2}+\cdots+\beta_{p} x_{i p}+\lambda
$$

\section{The Data}

The original dataset is taken from Gold sheet, the most famous handicapping resource for sport events in the United States. The NBA 2016-2017 odds and spreads dataset provides a wealth of information about win-loss records of NBA teams, including the historical performances and odds of all teams.

The dataset is organized by the alphabet order of each team with their full records and statistics. However, the organization of this dataset is not as straight forward as may be expected because each team played a different number of games in each year. The cleaning process starts with the removal of special characters and the identification of the data (Bhandari, Colet, Parker, et al., 1997). The results of this process are used to create a new dataset of NBA teams with distinct columns that define different attributes of the teams. Furthermore, the data cleaning step also separates meaningful information within one column.

The most significant characteristic of the dataset is the comprehensive amount of information found therein, from the proposed odds to actual game score and Over/Under odds. The dataset also details the spatial layout of the teams.

After the dataset has been well-validated and thoroughly cleaned, the next step 
is to create a data frame to explore statistical significance and then to build a statistical model to test the model. Every game appears twice in this data set, however, these are not independent rows, and we eliminate the duplicates by removing negative score differences (De Jonge \& Van Der Loo, 2013; Miljković, Gajić, Kovačević, \& Konjović, 2010).

Take a look of the first rows, we know that the dataset contain the informations of the games in the 2016-17 season such like date, team 1 and team 2, results, pointspread scores of the teams, score difference, site and overunder (See Figure 1).

We notice that in this dataset, this gives us the "symmetry" between score 1 and score 2. However, these are not independent rows, and before we model, we are going to remove the duplicates.

Because score difference comes from the difference between team 1 and team 2 , so we keep the positive ones so that we can remove the duplicates by filter the negative score difference, which should be 1309 rows.

\section{Explorations and Analysis}

\subsection{Model 1}

There are two different ways to measure how the odds by sportsbook align with the actual scores of the game: the first way is to compare Over/Under points against sportsbook odds and the second way is to compare the game score (or points spread) with sportsbook spread (odds). The two different ways make a comparison of betting odds with the actual score of the game.

We can model the total points based on team strengths (Manner, 2016). For example, if two "strong" teams play, both scores will be high, and the total will be higher, probably similar to the Over/under that is available prior to the start of the game. Then Now, we would like to model "team strength" with one coefficient per team, that could be used to predict future Over/Under values for games that have not yet been played. We can build a matrix for all 30 team, and use dummy variables then write a loop so that we can fill all games in that matrix.

We have the linear regression model:

$$
Y_{i}=\beta_{0}+\beta_{1} X_{1}+\beta_{2} X_{2}+\cdots+\beta_{30} X_{30}+i
$$

In which

$$
X_{1}, X_{2}, \cdots \text {, and } X_{30}
$$

\begin{tabular}{|c|c|c|c|c|c|c|c|c|c|c|}
\hline & dates & team1 & team2 & \multicolumn{2}{|c|}{ results pointspread } & score1 & score2 & diff & site & overunder \\
\hline 1 & $10 / 27$ & ATLANTA & WASHINGTON & W & -4 & 114 & 99 & -15 & $\mathrm{H}$ & 2020 \\
\hline 2 & $10 / 29$ & ATLANTA & PHILA. & W & -7.5 & 104 & 72 & -32 & V & $202 U$ \\
\hline 3 & $10 / 31$ & ATLANTA & SACRAMENTO & W & -7 & 106 & 95 & -11 & $\mathrm{H}$ & $203 U$ \\
\hline 4 & $11 / 2$ & ATLANTA & LA LAKERS & $\mathrm{L}$ & -12 & 116 & 123 & 7 & $\mathrm{H}$ & 2070 \\
\hline 5 & $11 / 4$ & ATLANTA & WASHINGTON & $\mathrm{L}$ & 1.5 & 92 & 95 & 3 & V & $206 U$ \\
\hline 6 & $11 / 5$ & ATLANTA & HOUSTON & W & -3 & 112 & 97 & -15 & $\mathrm{H}$ & $216 U$ \\
\hline
\end{tabular}

are dummy variables on 30 teams.

Figure 1. Preview of the games (first six rows). 
In the multiple regression model above, we ignore other non-dichotomous quantitative variables and another dichotomous quantitative variable which is home field advantage to estimate regression coefficients and to avoid the problems that this model would lead to (Roesser, 1975). For instance, if the home field advantage is to be used for an analysis of the relative performance of clubs, a different type of covariate would be required to account for the impact of home field advantage. Moreover, head-to-head performance differences at all levels of a competition would tend to further complicate the interpretation of the results, and would be difficult to adjust for, as the head-to-head comparisons would tend to provide incomplete answers to many of the questions that we want to determine. In fact, head-to-head performance differences arise due to interaction of variables, and it is conceivable that some of these could be eliminated through a more systematic test of potential statistical interactions between teams First we extract the total score from Over/Under.

Figure 2 shows the difference between the predicted value of total scores of 2 teams before the games and actual value of total scores of 2 teams. According to Figure 2, the range of actual score totals is wider than pre-game Over/Unders'. And the point approximation is symmetrical by $\mathrm{y}=\mathrm{X}$-ray, which is proved that the prediction is largely accurate.

Now build a matrix for all 30 teams, then use dummy variables. Then write a loop so that we can fill all the games in that matrix (Nasseri, Sohrabi, \& Ardil, 2008).

Figure 3 contains information about all 1309 games in that season. For example, on the first line, the value of "ATLANTA" and "LA LAKERS" is 1, which represents two teams of the first game.

Figure 4 shows that the coefficients of different teams from NBA are in the range of $90-120$, and most of teams' coefficients are near 105. HOUSTON, DENVER are the strongest teams in that season and DALAS, UTAH are the weakest teams in that season by virtue of coefficients.

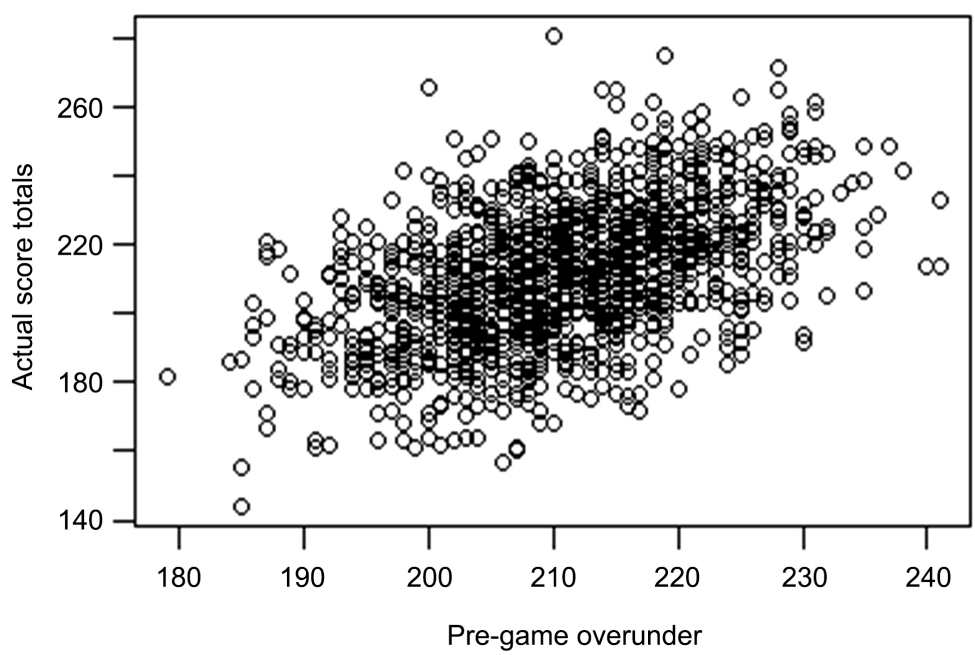

Figure 2. Scatter chart of pre-game Over/Under and actual score totals. 


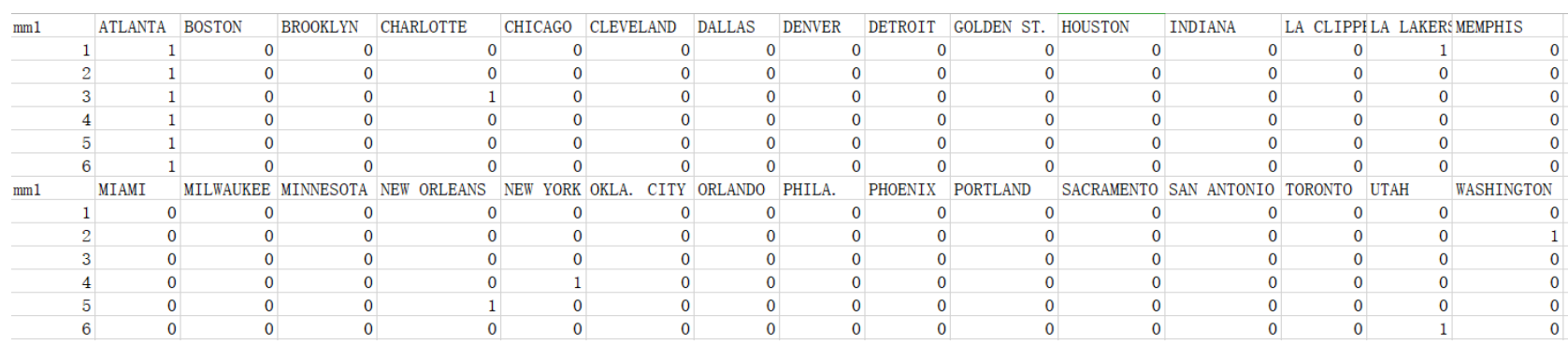

Figure 3. Part of the matrix 1 for model 1.

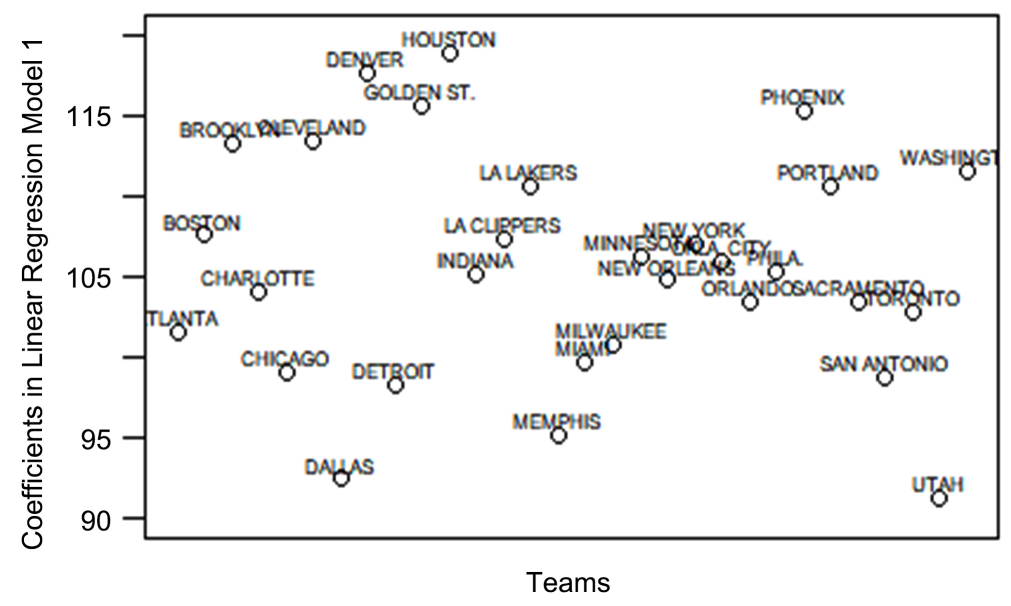

Figure 4. Distribution of value of coefficients of each teams.

The plot above shows the strength of each team in the NBA in the 2016-2017 season according to the number of points they scored. Indeed, when incorporating those coefficients into the model, we would get a partial picture of the strength of each NBA team. Using L.A. Lakers as the focal point, we want to compare the actual spread and the odds proposed by Vegas sportsbook.

\subsection{Model 2}

Now we are considering the model base on the difference between two teams. Since we filter the data set by keeping the games who have positive difference in score, then the actual difference should be all greater than 0 , and if the point spreads meet the true results, then most of point spreads should be greater than 0 .

Figure 5 shows that the actual score difference is in range of 0 - 50 (because the points which value of actual score difference is negative have been deleted all), and the value of difference from prediction is in range of (-20)-25. If the deleted data are involved in the scatter chart, the distribution of these remaining points and existing points are symmetry at the center of $(0,0)$.

Since we are interested in the points spread instead of Over/Under, if we were to select two different teams to compare, the coefficient of one team must be negative. Remember in the Over/Under linear regression model, there is no need to flip the sign of the coefficient of one team because we are interested in the total points of two teams. Simply choose L.A. Lakers as the primary predictor, we 
want to be able to show statistically that the point spreads of the Lakers in the 2016-2017 season compared to other teams. There are 3 different conditions of this model. LA. Lakers can be either team 1, team 2 or didn't take part in the game.

LA. Lakers is team 1:

$$
-\beta_{i}
$$

LA. Lakers is team 2:

$$
+\beta_{i}
$$

Lakers didn't take part in the game:

$$
\left(\beta_{\text {team1 }}-\beta_{\text {lakers }}\right)-\left(\beta_{\text {team2 }}-\beta_{\text {lakers }}\right)
$$

Remember the coefficients mean the strength difference between the team and Lakers.

For example: if the first team is 2 units better than Lakers and the other is 1 unit better, then we have $2-1=1$, that is: the first team is 1 unit better than the second, which meets what we expected.

We create another matrix in which L.A. Lakers is the selected team and compare its strength to the league then compare the predict score with the actual score.

Figure 6 " +1 " represent team 1 and " -1 " represent team 2.

Figure 7 shows the coefficients we got from model 2, for example, the coefficients for Houston is 12, which means it is 12 units better than Lakers.

Figure 8 shows that using past data and historical odds, the multiple linear regression model 1 can predict accurately up to $85 \%$ of the handicap odds of the game, and model 2 can predict $75 \%$. The multiple linear regression models are able to predict and take a correct bet on the final score, score difference and even the game outcome (Muske \& Rawlings, 1993).

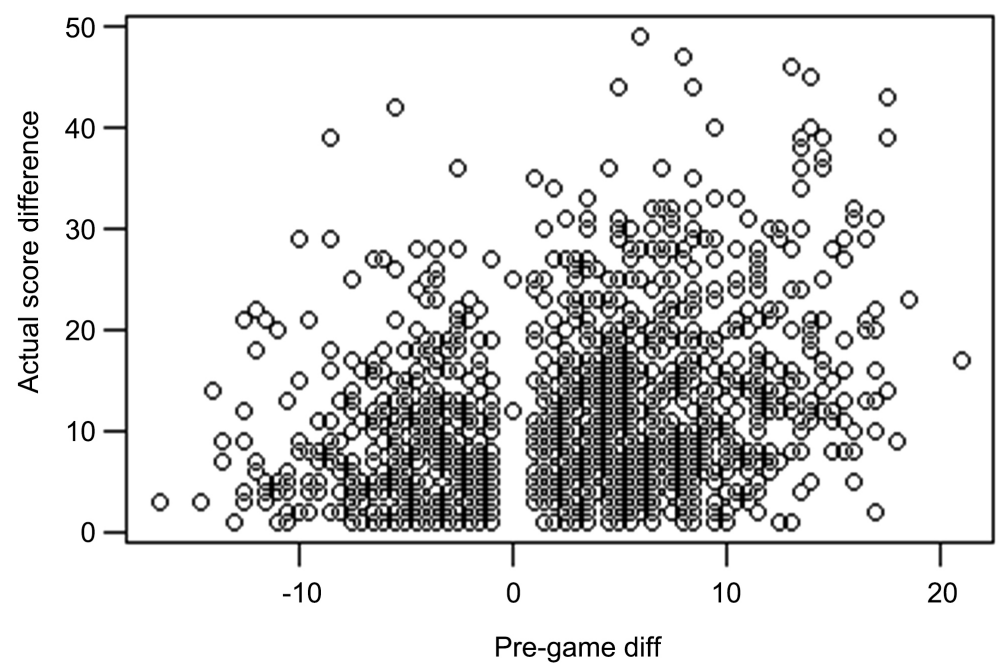

Figure 5. Scatter chart of pre-game difference and actual score difference. 


\begin{tabular}{|c|c|c|c|c|c|c|c|c|c|c|c|c|c|c|c|}
\hline $\mathrm{mm} 2$ & ATLANTA & BOSTON & BROC & OKLYN & CHARLOTTE & CHICAGO & CLEVELAND & DALLAS & DENVER & DETROIT & GOLDEN ST. & HOUSTON & INDIANA & LA CLIPPERS & MEMPHIS \\
\hline & 1 & 1 & 0 & 0 & 0 & 0 & 0 & & 0 & 0 & 0 & 0 & 0 & 0 & 0 \\
\hline & 2 & 1 & 0 & 0 & 0 & 0 & 0 & & 0 & 0 & 0 & 0 & 0 & 0 & 0 \\
\hline & 3 & 1 & 0 & 0 & -1 & 0 & 0 & & 0 & 0 & 0 & 0 & 0 & 0 & 0 \\
\hline & 4 & 1 & 0 & 0 & 0 & 0 & 0 & & 0 & 0 & 0 & 0 & 0 & 0 & 0 \\
\hline & 5 & 1 & 0 & 0 & 0 & 0 & 0 & & 0 & 0 & 0 & 0 & 0 & 0 & 0 \\
\hline & 6 & 1 & 0 & 0 & 0 & 0 & 0 & & 0 & 0 & 0 & 0 & 0 & 0 & 0 \\
\hline MIAMI & MILWAUKEE & MINNESOTA & A NEW & ORLEANS & NEW YORK & OKLA. CITY & ORLANDO & PHILA. & PHOENIX & PORTLAND & SACRAMENTO & SAN ANTONIO & TORONTO & UTAH & WASHINGTON \\
\hline & 0 & 0 & 0 & 0 & 0 & 0 & 0 & & 0 & 0 & 0 & 0 & 0 & 0 & 0 \\
\hline & 0 & 0 & 0 & 0 & 0 & 0 & 0 & & 0 & 0 & 0 & 0 & 0 & 0 & -1 \\
\hline & 0 & 0 & 0 & 0 & 0 & 0 & 0 & & 0 & 0 & 0 & 0 & 0 & 0 & 0 \\
\hline & 0 & 0 & 0 & 0 & -1 & 0 & 0 & & 0 & 0 & 0 & 0 & 0 & 0 & 0 \\
\hline & 0 & 0 & 0 & -1 & 0 & 0 & 0 & & 0 & 0 & 0 & 0 & 0 & 0 & 0 \\
\hline & 0 & 0 & 0 & 0 & 0 & 0 & 0 & & 0 & 0 & 0 & 0 & 0 & -1 & 0 \\
\hline
\end{tabular}

Figure 6. Part of the matrix 2 for model 2.

\begin{tabular}{|c|c|c|c|c|c|c|c|c|c|c|}
\hline & dates & team1 & team2 & results & pointspread & score 1 & score 2 & $\operatorname{diff}$ & site & overunder \\
\hline 1 & $10 / 27$ & ATLANTA & WASHINGTON & $W$ & -4 & 114 & 99 & -15 & $\mathrm{H}$ & 2020 \\
\hline 2 & $10 / 29$ & ATLANTA & PHILA. & $W$ & -7.5 & 104 & 72 & -32 & V & $202 \mathrm{U}$ \\
\hline 3 & $10 / 31$ & ATLANTA & SACRAMENTO & $W$ & -7 & 106 & 95 & -11 & $\mathrm{H}$ & $203 \mathrm{U}$ \\
\hline 4 & $11 / 2$ & ATLANTA & LA LAKERS & $\mathrm{L}$ & -12 & 116 & 123 & 7 & $\mathrm{H}$ & 2070 \\
\hline 5 & $11 / 4$ & ATLANTA & WASHINGTON & $\mathrm{L}$ & 1.5 & 92 & 95 & 3 & V & $206 \mathrm{U}$ \\
\hline 6 & $11 / 5$ & ATLANTA & HOUSTON & $W$ & -3 & 112 & 97 & -15 & $\mathrm{H}$ & $216 \mathrm{U}$ \\
\hline
\end{tabular}

Figure 7. Team strength compared with LA. Lakers.

\begin{tabular}{|r|r|}
\hline$x 1$ & $x 2$ \\
\hline 1.000000 & 0.859389 \\
\hline 0.859389 & 1.000000 \\
\hline$x 1$ & $\times 2$ \\
\hline 1.000000 & 0.752280 \\
\hline 0.752280 & 1.000000 \\
\hline
\end{tabular}

Figure 8. Compared predicted score with actual score.

\section{Conclusion}

The prediction algorithm can be improved by incorporating home field advantage, and other important factors on player performance ensure a more reliable and accurate prediction, for example, including three-point shooting percentage, the number of rebounds, and more detailed offensive or defensive data and finding coefficients for how each variable that affects the result. We can even collect the individual players' data so that when there are personnel adjustments between teams, we can get a new model of team strength. Furthermore, a head-to-head analysis would provide insight into the interactions among different factors affecting performance and what would be the best way to combine different variables into the predictive power of the predictive algorithm. For example, while Houston Rockets players score more points than the other teams, their small-ball style of play generates a high percentage of turnovers, which can also lead to low quality offensive performance against teams with stellar defenses such as the Spurs. These observations motivated us to study different ways of predicting team strengths by combining these different elements of the team and individuals' performances. Moreover, another method of analyzing the game of basketball in aggregate is to look at the designated odds and the corresponding probability of different teams' matches. However, this method is often hampered 
by the complex structure of the game and players' tendencies and strategies.

For R code regarding to this project, see Appendix I.

\section{Conflicts of Interest}

The authors declare no conflicts of interest regarding the publication of this paper.

\section{References}

Bhandari, I., Colet, E., Parker, J., et al. (1997). Advanced Scout: Data Mining and Knowledge Discovery in NBA Data. Data Mining and Knowledge Discovery 1, 121-125. https://doi.org/10.1023/A:1009782106822

De Jonge, E., \& Van Der Loo, M. (2013). An Introduction to Data Cleaning with $R$. The Hague: Statistics Netherlands.

Golec, J., \& Tamarkin, M. (1995). Do Bettors Prefer Long Shots Because They Are Risk-Lovers, or Are They Just Overconfident? Journal of Risk and Uncertainty, 11, 51-64. https://doi.org/10.1007/BF01132730

Humphreys, B. R., Paul, R. J., \& Weinbach, A. P. (2013). Consumption Benefits and Gambling: Evidence from the NCAA Basketball Betting Market. Journal of Economic Psychology, 39, 376-386. https://doi.org/10.1016/j.joep.2013.05.010

Manner, H. (2016). Modeling and Forecasting the Outcomes of NBA Basketball Games. Journal of Quantitative Analysis in Sports, 12, 31-41. https://doi.org/10.1515/jqas-2015-0088

Miljković, D., Gajić, L., Kovačević, A., \& Konjović, Z. (2010). The Use of Data Mining for Basketball Matches Outcomes Prediction. IEEE 8th International Symposium on Intelligent Systems and Informatics, Subotica, 10-11 September 2010, 309-312. Ahttps://doi.org/10.1109/SISY.2010.5647440

Muske, K. R., \& Rawlings, J. B. (1993). Model Predictive Control with Linear Models. AIChE Journal, 39, 262-287. https://doi.org/10.1002/aic.690390208

Nasseri, H., Sohrabi, M., \& Ardil, E. (2008). Solving Fully Fuzzy Linear Systems by Use of a Certain Decomposition of the Coefficient Matrix. Int. J. Comput. Math. Sci, 3.

Roesser, R. (1975). A Discrete State-Space Model for Linear Image Processing. IEEE Transactions on Automatic Control, 20, 1-10. https://doi.org/10.1109/TAC.1975.1100844

Spann, M., \& Skiera, B. (2009). Sports Forecasting: A Comparison of the Forecast Accuracy of Prediction Markets, Betting Odds and Tipsters. Journal of Forecasting, 28, 55-72. https://doi.org/10.1002/for.1091 
Appendix I: R code

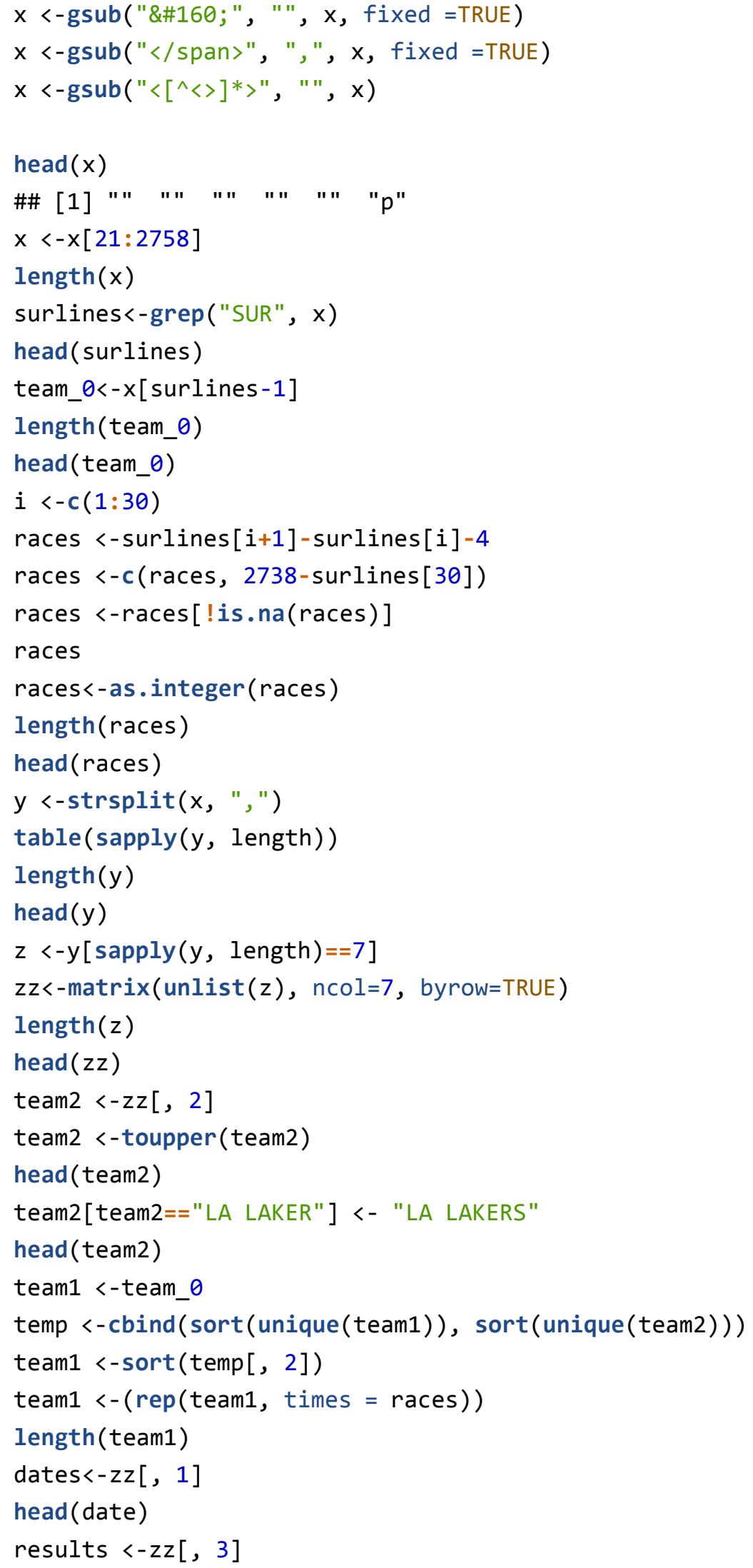




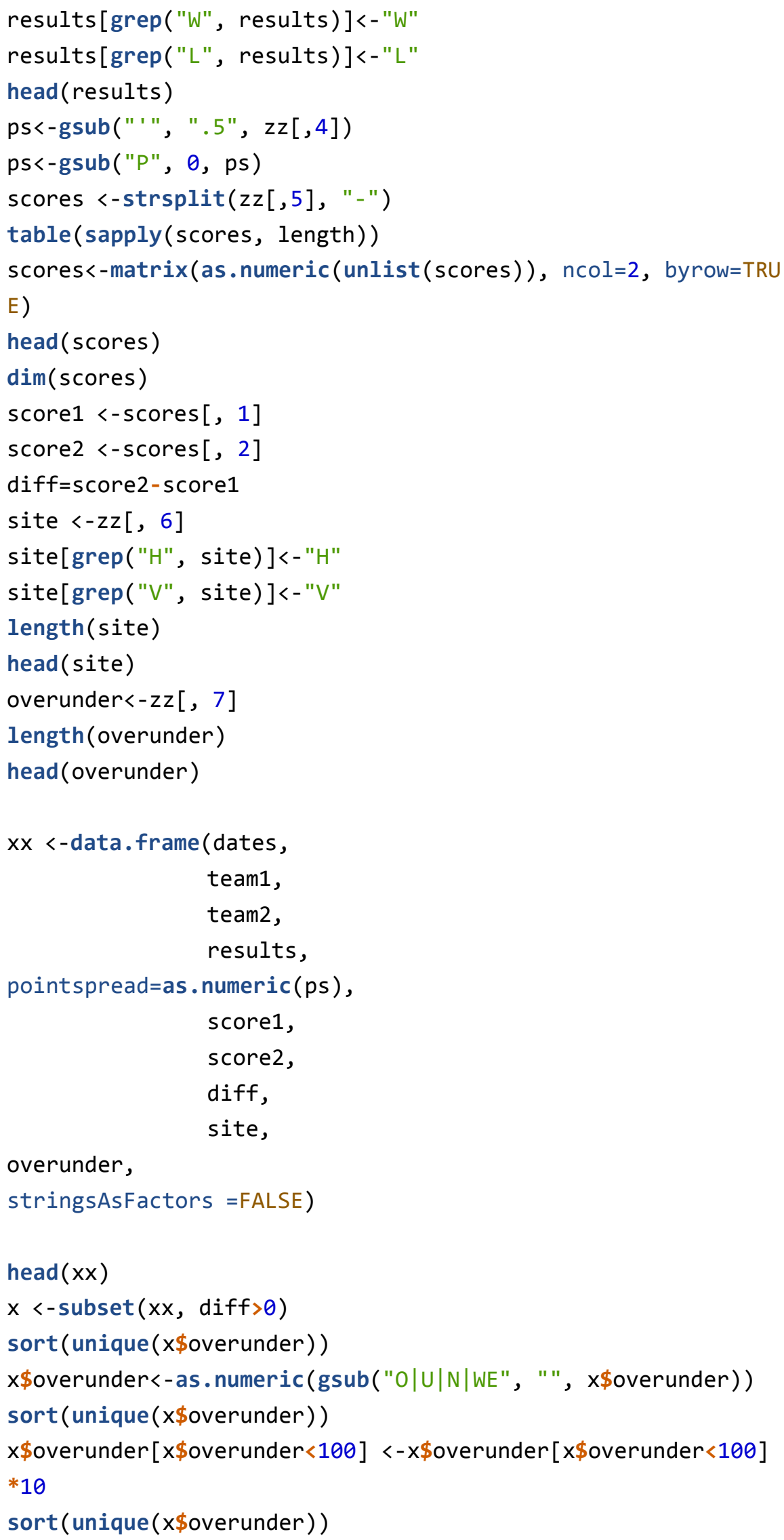




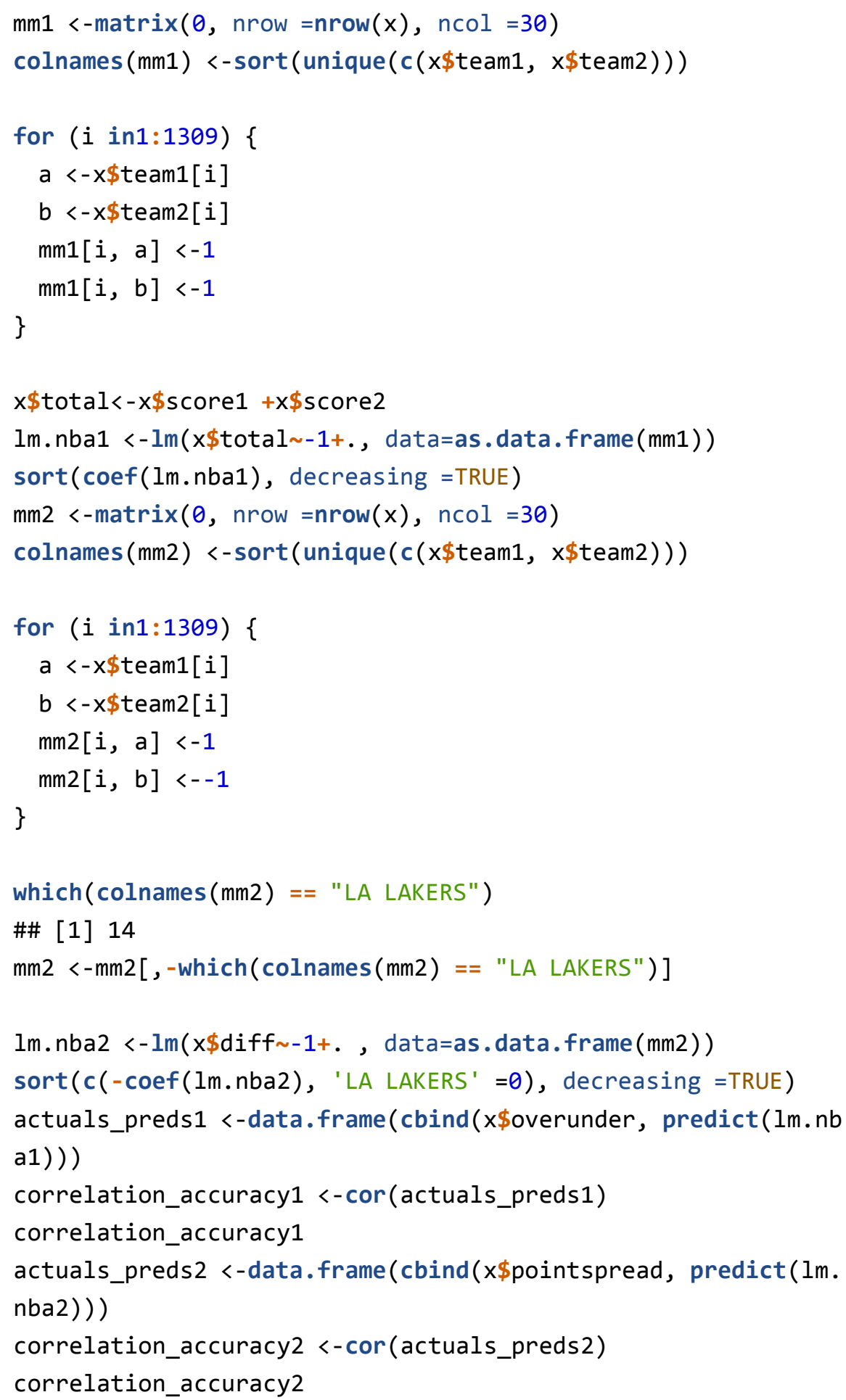

